\title{
Intelligent Building Security System Design based on Internet of Things Technology
}

\author{
ZhangBo \\ Hainan Vocational College Of Political Science And Law
}

Keywords: Internet technology; Intelligent buildings; Security; Design

\begin{abstract}
Network security system constructs unified management and communication software under the monitor of alarm management center to make the distribution network between each subsystem and central monitoring equipment. Under the control of alarm management center and central management, achieve automation management and realize the integration of equipment and software and hardware, etc, so as to promote information integration and control. Based on Internet of things technology, the paper mainly analyzes the security system design of intelligent buildings.

At present, architectural design lacks intelligent building mechanical and electrical products which lead to low level of industrialization, low product quality, and the situation of poor application effect [1]. In the subsequent installation of intelligent building, when installation personnel use mechanical and electrical equipment, there are a lot of problems, which mainly because the installation is not in accordance with the requirements [2]. To solve these problems, based on Internet of things technology, the article designs safe guard system in intelligent buildings, combines with the actual needs of users, formulates the all-round, three-dimensional safe guard system design plan in intelligent building according to security technology development direction, in order to form complete system based on the current technology, and widely use it in building design.
\end{abstract}

\section{THE DEFINITION OF SAFE GUARD SYSTEM}

In domestic intelligent architecture, many experts have reached a consensus of understanding that construction safety and protection system is taking building as a carrier to realize centralized regulation. Integrate all safety devices and systems together through forms of collection or combination, and form a comprehensive security system. Combined with the actual demand, realize autonomic running or integrate with some automatic systems. Construction safety and protection system has experienced a structural transformation from simple to complex, which is also a structure shift from scattering to combination. This changes the original simple electronic anti-theft alarm system. Under the support of Internet technology, we formed an alarm, monitoring and networking system. Through development, a comprehensive monitoring system with final in and out monitoring and video monitoring is formed. Currently, there is an integrated safety system with security patrol and anti-burglar alarm integration in intelligent building. The specific safety guard system is as follows:

In integrated security technology prevention's comprehensive management system, each subsystem is respectively set up with the help of common communication platform and management software to realize the automation of the whole system regulation. The development of the system is quickly. Given the limitations of consumption and technical level, it is rarely used in the mainland. It is popular in foreign and coastal high-grade office buildings. In the above three modes, the last security system is the future development trend. At present, the developing integration model is at a high level. With the development of science and technology, system integration's wide degree is also gradually improving.

In a loose type safety system, each subsystem alone sets and runs to make the simple linkage. This system was prevailed in the 1990s, and many buildings used this device. Although without higher level of technology, after modification, it can have a longer life cycle. At present, most cities' Internet centers carry out basic construction on the basis of distributed security system by using 
some network connection system. Such as public telephone network, it installs different kinds of anti-theft alarm devices for customers, and forms the security network system. Security alarm system centralized control users' safe guard system reduces the cost of overhead and prompts the safe operation of social public security system.

In modular safety integrated management system, each subsystem does independent setting. By using special software and communication interface, assemble each subsystem to realize the centralized control of the whole system. This is the integrated management system with safety and defense. we often use these systems such as video monitoring system and security system, etc. In recent years, most residential buildings and office buildings are using them, and the technology and products are more and more perfect.

\section{SECURITY SYSTEM ARCHITECTURE}

Security system architecture and OSI layered structure are similar. Detector and front-end equipment generally realize signal transmission via physical layer and field controller. Controller covers different hierarchy of needs, however, data link layer and physical layer are needed. Controller realizes digital transformation of detector signals to provide control mechanism service for signal transmission differential and transmit probe itself and the detected signal to the upper machine in central control room or police computing room. Upper machine in general is a personal computer or industrial PC, etc. Upper body includes data link layer, application layer and transport layer, etc., which presents different alarm forms and easy signal transmission. Send to the monitoring personnel in form of image and voice in multimedia technology. In addition, it still can form linkage and manipulation with other systems. Between the upper and lower planes, there are a variety of communication ways, such as BACnet or TCP/IP protocol. In addition, S - 485 or CAN bus way can also be used. And of course, coaxial cable, twisted pair and so on can also be used. In fact, the match between higher and lower is changing, which depends on some objective factors, such as hardware, software and equipment, etc.

\section{TECHNOLOGY SECURITY SYSTEM WITH ELECTRONIC SECURITY}

Technology security system brings together a variety of applications, such as biology and chemistry. With the rapid development of electronic information technology, technology security system gradually turns into a security system with electronic prevention as the main form. Building security system mainly includes automobile library integrated management system and burglar (rob) alarm subsystem, etc. These systems alone set up and run with central system centralized control, and can also cooperate with other centralized system.

In TV monitoring alarm subsystem, closed-circuit television monitoring alarm system is usually applied to the building and the surroundings' key public area to achieve real-time monitoring of the images and review. System front-end is configured a variety of cameras types and ancillary equipment, usually with optical transmission or coaxial cable transmission. System terminal equipment, usually with separate monitor alarm center console or video monitoring center console, is used to display and record, etc. Closed circuit television monitoring system, access control system and usually anti-burglar alarm system for safety use are under central control of safe guard system. Separate operation of closed circuit television monitoring system can realize picture editor and switch and show computer specific information, such as time and date. It can automatically switch the specified scene to a monitor, in addition, it also can carry on long time monitoring of key parts. The system realizes connection on the set with 110 turning center networking. When the alarm occurs, the system performs processing and confirmation of the sound and image in a timely manner. This is a function of safety and protection system. It can run independently and perform linkage system, or work in combination with other systems to ensure the accuracy of the alarm.

The stolen (rob) alarm subsystem is divided as space protection and perimeter protection, etc., and usually the system front-end is configured different types of alarm detector. We can use wired and wireless transmission mode. Wired transmission is divided into telephone line transmission and 
special line transmission. System terminal is used to display and communication with the use of a separate alarm controller or alarm center console. No matter what way, ensure that in the event of illegal invasion, accurately without error do alarm and review. Leakage alarm is banned, and false alarms should be in the acceptable range. To prevent robbery, the system set up the interface with 110 turning center network.

In the gateway control alarm subsystem, gateway control alarm system uses advanced electronic information technology to permit or prohibit the entering command for the pedestrians outside a building. It is an automated electronic system with the integration of record and report to the police, usually consisting three parts such as access control execution system and target recognition system, etc.

\section{HOME ALARM SYSTEM}

Alarm system set up different types of front detector within the scope of specific space to run information in the channel, and submit it to the back end equipment to achieve the purpose of alarm or control on the spot. Alarm system has the following several types: emergency alarm, illegal intrusion alarm and general fire alarm, etc. General residential alarm satisfies a wide range of practicality and system correlation. The broad practicability can protect every household in the district and the guard system is in control, which can timely report to the police. System correlation mainly refers to the correlation of entrance guard system and monitoring system to optimize the network.

\section{A. Alarm system architecture}

The concept of alarm system structure is in change at any time, and it has close contact with the development of alarm system. The alarm system development has experienced the transition from traditional single alarm device model to new model with Internet configuration, which realizes the simultaneous monitoring of residential areas, and integrates parking lot and entrance control system together, and forms a joint protection system. The current cities use this system model. Inherent phone Internet system is a backward system with slow reaction speed, high cost, and less stability. It will be gradually replaced by Internet monitoring system, which implements network correlation within the region with central processor and many detectors, etc. In accordance with the agreement, send out alarm information. It is an independent, efficient, and economical system model. Many current residential alarm systems use RS - 485 bus, and some use Lon Works or the CAN field bus. The former system bus has such characters as simple operation and low cost, however, under the background of social network, based on field bus CAN in TCP/IP protocol, home alarm system advantage is still outstanding. Networking type residential alarm system includes four aspects, and underlying front end detector is one of them. In addition, there are upper networking alarm management center and lower house alarm control unit, etc. Bottom and lower layer communication modes are: wireless communication, bus communication and telephone communication. When the lower level information goes through the residence, communicate via Ethernet with the remote query terminal and the help of the Internet, and connect the residential local area network and lower controller for communication.

\section{B. Networking type residential alarm system design}

Home alarm system makes judgments through feelings outside changes such as sound, light, electric, magnetic got by a variety of front-end probe, and finally sends alarm message. Alarm controller can master monitoring status of each detector in time. In dynamic control alarm, pack the alarm message into packets in accordance with the agreement, and send them to residential alarm management center. In addition, support HTTPWeb service. In network alarm management center's upper center management, alarm information processing and database are two constituent parts. Information processing center makes communication protocol interpretation, and present the alarm information collected in the form of alarm. Alarm information database contains systematic alarm data and basic configuration information to register alarm and police information, provide alarm information query function and perform alarm information management. Community security personnel, according to the alarm information, quickly capture the status of emergency alarm and 
take effective safety precautions to provide security for the living people. Using the Internet, realize the alarm information query and focus on family's security status through remote log in end. Remote query terminal has not high requirements for the configuration of Web browser, and can layout or evacuate alarm communication host in the form of calling.

Engineering design uses alarm communication way for professional bus system alarm. Combined with the feature of RS - 485 buses, the alarm system lacks integration, real-time and flexibility. The design uses IP network alarm system design as reference, effectively uses the characteristics of multi-protocol total capacity and integrated wiring, and makes intelligent and digital Internet advanced advantage into full play. Family host anti-theft alarm communication uses RS - 485 bus and network connection. RS - 485 buses wire all uses specification 2 x $1.0 \mathrm{~mm}$ shielded twisted-pair cable for wiring work. Single RS - 485 bus wiring should prevent branch wiring. Multiple RS - 485 forms CAN bus after the transformation of MTGW bus conversion module, and CAN bus is connected to alarm receiving host. Receiver CAN bus uses good performance $2 \times 1.5 \mathrm{~mm}$ shielded twisted-pair cable, and each MTGW is configured at most 120 RS - 485terminal equipment. The bus uses star connection. The power cord usually uses RVV - 2-1.5 two core sheathed wires for corridor lights, and four core wires are used from the corridor to residents. Combine all source detector number, each 12v2a dc power supplies for 6-10 households use service. Anti-theft alarm communication host transmit a variety of alarm information received by the main bus to residential alarm receiving host. Community networking alarm management center, combined with address coding information displayed on anti-theft alarm communication host, identifies the corresponding building number and positions the alerting users accurately. Present the alarm type through alarm communication post alarm information, make rapid alert disposal, and prevent early-warning review timeout.

\section{CONCLUSION}

Intelligent construction installation is a trend of future development in construction. In house construction, intelligent building has become the mainstream in the future. Intelligent building saves energy consumption, protects environment, and is efficient, comfortable and so on. In both scientific research and management and training staff, develop detailed specification to ensure the dynamic control. Better play intelligent building's function and provide high quality living environment [3]. Internet of things technology is applied to intelligent buildings public security systems, sets up a platform of regulation and linkage, reduces monitoring cost overhead, and realizes the high quality of supervision and emergency linkage [4]. Based on Internet of things technology, intelligent building security system design is the inevitable direction of the future architectural design.

\section{REFERENCES}

[1] Liu Qing. Intelligent building mechanical and electrical installation quality control [J]. Technology Entrepreneurship Monthly, 2014, (6) : 177-178.

[2] Huang Dechao. Intelligent building mechanical and electrical installation quality control [J]. Journal of Urban Geography, 2015, (14) : 286-287.

[3] Zheng Wei. Intelligent building mechanical and electrical installation quality control research [J]. Journal of Wireless Technology, 2015, (03) : 121-122.

[4] Wang Yao. Internet of things technology application research in intelligent building public security system [D]. Chang 'an University, 2013. 